\title{
Specification of Forces in Rotational Separator
}

\author{
Marlena A. Gronowska \\ Gdańsk University of Technology, Faculty of Civil and Environmental Engineering, ul. Narutowicza 11/12, \\ 80-233 Gdańsk, Poland, e-mail: marlena.gronowska@wilis.pg.gda.pl
}

(Received December 12, 2011; revised March 22, 2012 )

\begin{abstract}
Today, intensive urban development calls for new technologies in the field of environmental engineering. Extensive infrastructure requires more roads and motorways, as well as larger areas covered with concrete, which altogether leads to greater volumes of water creating surface run-off. Rotational separators, besides lamella and coalescence units, may provide a highly efficient method for treatment of storm water running off roads and motorways. This paper is focused on two important aspects of the operation of rotational separators: forces acting on a particle in liquid flow, and pressure distribution in the chamber. The resulting relations constitute crucial elements in the future method of designing such devices.
\end{abstract}

Key words: centrifugal force, rotational separator, sedimentation, storm water, waste water treatment

\section{Notation}

A $\quad-$ semi-empirical multiplier

$a_{r} \quad-$ associated mass coefficient,

$C, C_{1}, C_{2}, D$ - integration constants,

$C_{D} \quad-$ drag coefficient,

$d_{i n} \quad-$ inlet diameter,

$d_{p} \quad-$ particle diameter,

F $\quad-$ force,

$g \quad-$ gravity acceleration,

$H \quad-$ water depth,

$\mathbf{i}, \mathbf{k} \quad-$ radial and vertical versors,

$m_{p} \quad-$ particle mass,

$p \quad-$ pressure,

$Q \quad-$ discharge,

$r, \phi, z \quad-$ cylindrical coordinates,

$R \quad-\quad$ radius of separator chamber,

$S_{c} \quad-$ cross-sectional area of particle, 


$\begin{array}{ll}\mathbf{u} & - \text { fluid velocity, } \\ \mathbf{v}_{\mathbf{p}} & - \text { particle velocity, } \\ V & - \text { particle volume } \\ \mu & - \text { dynamic viscosity coefficient, } \\ \rho & - \text { density of liquid, } \\ \rho_{p} & - \text { density of particle, } \\ \omega & - \text { angular velocity. }\end{array}$

\section{Introduction}

\subsection{The Need for Storm Water Treatment}

Residential storm water constitutes a separate category of sewage that has to be adequately handled. Storm water should be collected, transported and treated according to legal requirements, to be finally discharged into a receiver, e.g. a river, drainage basin, or groundwater flow. Main pollutants present in water from atmospheric precipitation (rain and snow) include suspended matter and petroleum derivatives, as well as heavy metals.

According to the Polish Standard PN-S-02204:1997, the maximum allowable concentration of suspended solids is $50 \mathrm{mg} / \mathrm{dm}^{3}$. In Poland, research on the concentrations of contaminants in storm water (Osmólska-Mróz 1996) indicate that for a daily traffic flow of 12000 vehicles the amount of suspended matter is $292 \mathrm{~g} / \mathrm{m}^{3}$. This means that in the case of a road section $1000 \mathrm{~m}$ long and $50 \mathrm{~m}$ wide, assuming an annual rainfall of $600 \mathrm{~mm}$, the mean load of suspended matter is circa $24 \mathrm{~kg} /$ day - approximately one bucket of sand is collected from such a road section every day.

With the allowable limit for concentration of suspended matter set at $50 \mathrm{mg} / \mathrm{dm}^{3}$, storm water should be treated to contain no more than $4.12 \mathrm{~kg} /$ day of suspended matter for the road section under consideration. The amount of suspended matter in storm water greatly exceeds the maximum allowable value, and so there is a strong need for wastewater treatment installations.

A technical solution to the problem of contamination includes a system for a direct treatment of storm water before discharge. According to the Polish Standard PN-S-02204:1997, expressways and motorways require a drainage system that includes four elements: collection of rainwater flowing down the road, removal of water from the road, water purification, and water disposal into the environment.

\subsection{Types of Separators}

Separators constitute a special category of devices (e.g. grit chambers, clarifying tanks or settling tanks) employed for the gravitational removal of suspensions from liquids. Arranged along roads (well beyond urban areas), these devices may be successfully used for storm water treatment on a local scale. Separators are required to be compact and efficient, as well as fracture-proof and durable. In order to meet these 
standards, such devices are equipped with additional subsystems enhancing the process of separation. Lamella separators, including an additional lamellar pack of plates, are designed to separate both suspended matter and petroleum derivatives. In coalescence separators, liquid flow is directed through a cylindrical filter insert that removes oil from wastewater. The design of a rotational separator (tangential inlet pipe) forces the flow of the liquid to be circular (Fig. 1a). As a result, by rotating around a vertical axis, liquid flow generates a centrifugal force that acts on every single suspended particle by retarding its motion. Particle detention time within the device is prolonged, which increases the probability of the particle being separated from the feed liquid stream in the process of sedimentation or flotation. The principle of its operation is analogical to that of the dust removal process in a cyclone (Fig. 1b), in which the centrifugal force generated by a rotating gas stream pushes dust particles towards the outside wall of the cyclone. Particles approach this wall, moving towards the bottom of the object owing to the action of the gravity force.

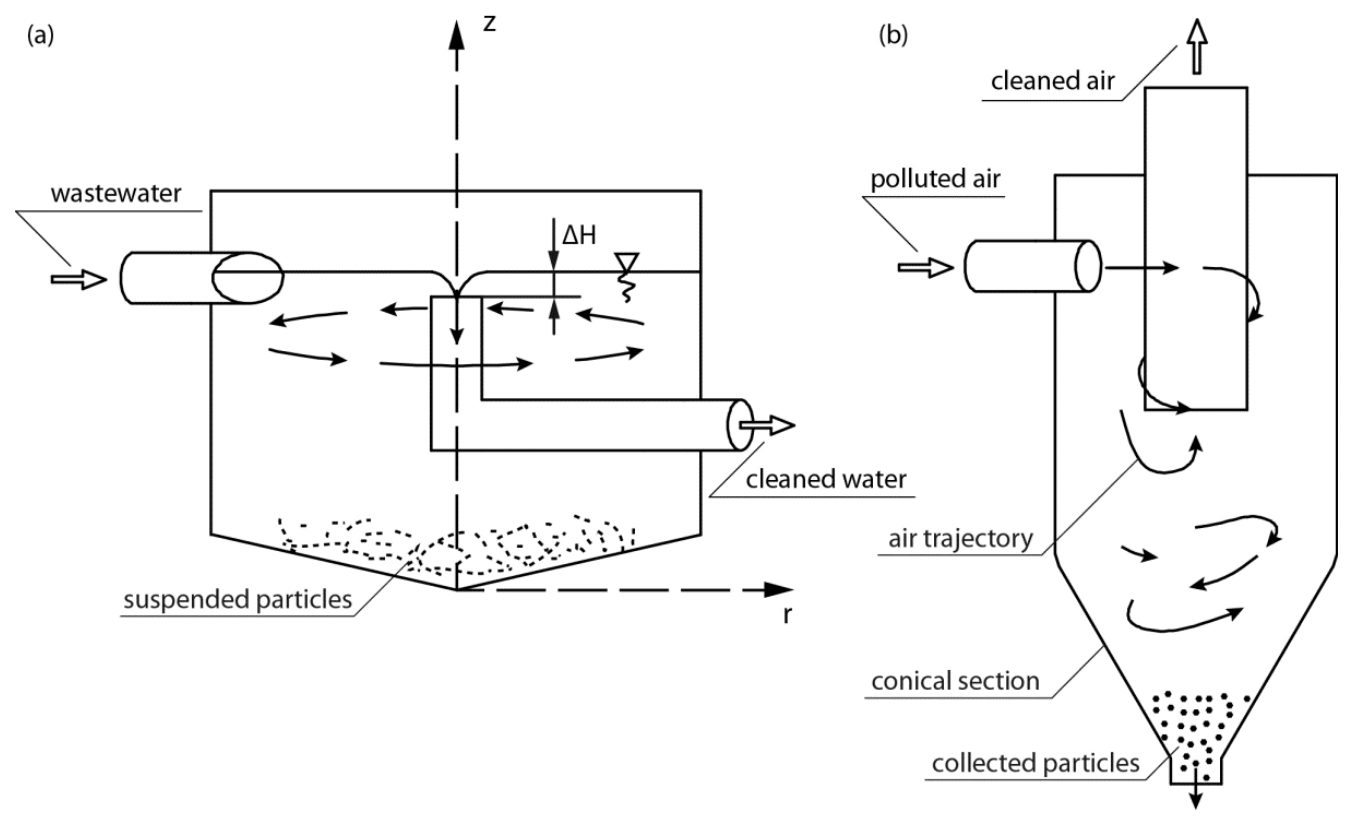

Fig. 1. Schematic diagrams of the main types of circulative separators: a) rotational separator; b) cyclone

\section{Rotational Separators Design}

\subsection{General Remarks}

Producers of devices for local wastewater treatment are becoming increasingly interested in rotational separators. In order to respond to the growing need for an efficient 
method of designing separators, a revision of the methods (developed with an emphasis on cyclones) found in the literature on wastewater treatment was performed (Bernardo et al 2007, Smith 1959, Trawinski 1969, Warych 1998).

\subsection{Forces Acting on a Particle}

The fundamental element of every separator design method is the analysis of forces acting upon a representative particle suspended in a fluid flow (Fig. 2). Considering the problem, it is convenient to divide forces acting on a particle into two categories:

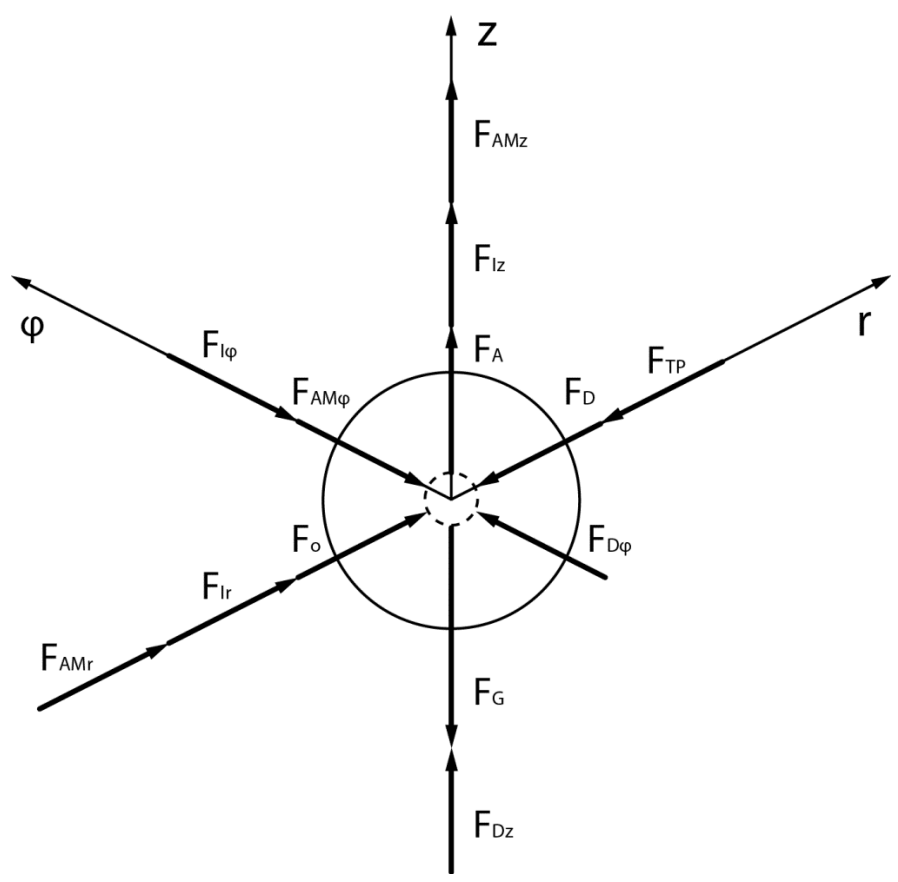

Fig. 2. Forces acting on a particle in fluid

- "classical" forces described by well-investigated relations (Soo 1996):

- the drag force which can be expressed by the Stoke's formula (laminar case):

$$
\mathbf{F}_{\mathbf{D}}=\mathbf{F}_{\mathbf{D S}}=3 \pi \mu d_{p}\left(\mathbf{u}-\mathbf{v}_{\mathbf{p}}\right),
$$

or by the Newton's formula (turbulent case):

$$
\mathbf{F}_{\mathbf{D}}=\mathbf{F}_{\mathbf{D N}}=C_{D} S_{c} \frac{\rho\left|\mathbf{u}-\mathbf{v}_{\mathbf{p}}\right|\left(\mathbf{u}-\mathbf{v}_{\mathbf{p}}\right)}{2},
$$

- the force of inertia: 


$$
\mathbf{F}_{\mathbf{1}}=\rho_{p} V \frac{d \mathbf{v}_{\mathbf{p}}}{d t}
$$

- the associated mass force:

$$
\mathbf{F}_{\mathbf{A M}}=a_{r} \rho V \frac{d\left(\mathbf{u}-\mathbf{v}_{\mathbf{p}}\right)}{d t}
$$

as well as known factors acting vertically: the gravity force $\mathbf{F}_{\mathbf{G}}$ and the hydrostatic lift $\mathbf{F}_{\mathbf{A}}$.

- "new" forces, without standard description for rotational separators, acting radially:

- the centrifugal force (Stairmand 1951):

$$
\mathbf{F}_{\mathbf{0}}=m_{p} \omega^{2} r \mathbf{i}=m_{p} \frac{u_{t}^{2}}{r} \mathbf{i}
$$

- the pressure force called the transversal pressure effect or the "buoyancy force": (Soo 1996):

$$
\mathbf{F}_{\mathbf{T P}}=-V \frac{d p}{d r} \mathbf{i} .
$$

The mathematical description of rotational separator operation is based on the equation of trajectory of a moving particle:

$$
\frac{d \mathbf{r}_{p}}{d t}=\mathbf{v}_{\mathbf{p}}
$$

Assuming that the particle velocity $\mathbf{v}_{\mathbf{p}}$ is known, the solution of Eq. (7) provides the shape of the trajectory expressed by the radius vector $\mathbf{r}_{p}(t)$. The function describing the particle velocity $\mathbf{v}_{\mathbf{p}}$ is a solution of Newton's second law equation (Soo 1996). An effective balance of the abovementioned forces depends on the direction of fluid motion. Vertically, the following forces are taken into account:

$$
\rho_{p} V \frac{d v_{p z}}{d t}-a_{r} \rho V \frac{d\left(u_{z}-v_{p z}\right)}{d t}=\mathbf{F}_{\mathbf{D Z}}-\mathbf{F}_{\mathbf{G}}+\mathbf{F}_{\mathbf{A}},
$$

whereas for the balances of horizontal (radial and circumferential) forces, key relations in the separator dimensioning process have the form:

$$
\begin{gathered}
\rho_{p} V \frac{d v_{p r}}{d t}-a_{r} \rho V \frac{d\left(u_{r}-v_{p r}\right)}{d t}=\mathbf{F}_{0}-\mathbf{F}_{\mathbf{D r}}+\mathbf{F}_{\mathbf{T P}}, \\
\rho_{p} V \frac{d v_{p \varphi}}{d t}-a_{r} \rho V \frac{d\left(u_{\varphi}-v_{p \varphi}\right)}{d t}=\mathbf{F}_{\mathbf{D} \varphi} .
\end{gathered}
$$

In such a situation, each and every suspended particle is treated as a material volume of specific mass and geometrical parameters, and its individual shape is included 
in the drag force coefficient $C_{D}$ (Eq. (2)). Shapes of particles of suspended solids removed in rotational separators are regular to such extent that can be assumed to be spherical.

While describing motion of suspension, an important aspect of influence of neighbouring particles on each other should be taken into account. Interaction between particles can be neglected when volume concentration of particles does not exceed $1 \%$. In case of storm waste water, for which rotational separators are designed, maximum concentration of suspension reaches $26 \mathrm{~kg} / \mathrm{m}^{3}$ (Osmólska-Mróz 1996). With suspension density on the level of $2.7 \mathrm{~kg} / \mathrm{m}^{3}$, maximum volume is $9.63 \mathrm{dm} / \mathrm{m}^{3}$, so volume concentration is $0.963 \%<1 \%$.

As already stated, particle trajectory $\mathbf{r}_{\mathbf{p}}(t)$, being the solution of system of equations (Eqs. $(7,8,9,10))$, yields the fullest image of separator operation. In order to acquire the solution, fluid flow velocity field, of $u_{r}, u_{\varphi}, u_{z}$ components, needs to be determined first. In general case, velocity field, and then suspended particle trajectory, are found by numerically solving equations governing fluid flow (e.g. Bernardo et al 2007). As particles forming the suspension are almost unlimited in number, scope of the calculus should be reduced to characteristic ones only (chosen in terms of their size and initial position).

Despite being general and detailed at the same time, such an approach could hardly constitute a fundamental tool in engineer's everyday work. While designing technical objects, applied mathematical relations are desired to be formally simple. Algebraic expressions that allow for elastic calculation of needed values are especially useful. Methods employing differential equations (physically more detailed) lack this elasticity (widely known difficulty in solving inverse problems). The need for formally simple methods of rotational separators design constitutes the driving factor for further research.

Practical Separator design methods found in literature were developed for two characteristic states of the object under consideration:

- the critical particle (the smallest of the particles to be removed by separation) is motionless relative to the horizontal plane,

- the critical particle is moving with the flowing fluid.

In the case of rotational separators, both methods have already been discussed in detail (Gronowska, Sawicki 2011). The physical analysis of the description of forces in both methods indicates the presence of some errors. First of all, the Stoke's formula for the stress force (Eq. (1)) should be replaced by the Newton's formula (Eq. (2)) as the process of the separation of suspension occurs in turbulent flow conditions. Furthermore, tangential velocity distribution applied in both methods corresponds to fluid motion in a centrifuge - a cylinder rotating around a vertical axis (Fig. 3a) - instead of cyclones and rotational separators, in which the chamber is fixed and circular motion is generated by a tangential inlet pipe (Fig. 3b). 
(a)

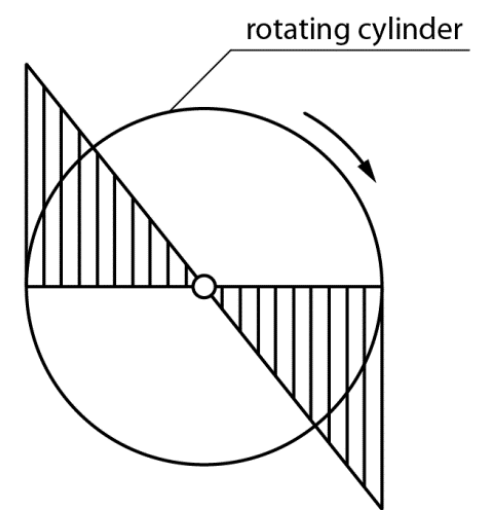

(b)

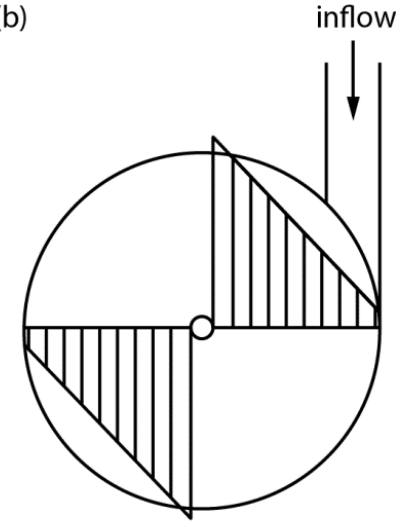

Fig. 3. Tangential velocity fields in: a) centrifuge, b) circulative separator

\subsection{Velocity Distribution in a Rotational Separator}

In order to determine the actual tangential velocity distribution, a laboratory model of a rotational separator was constructed (Fig. 4). Point measurements of the flow velocity were performed with a micro-propeller current meter. Sample results are presented in Fig. 5 (tangential velocity) and Fig. 6 (radial velocity). Considering the results and physical conditions, it was assumed that the tangential velocity profile in a rotational separator may be approximated by a linear function. Moreover, measurement results indicate that vertical tangential velocity profile is quite uniform, what allows for assumption that tangential velocity is a function of radius $r$ only:

$$
u_{t}(r)=A(R-r)
$$

where multiplier $A$ has been derived from the condition of energy flux balance which states that energy flux delivered to object's chamber by tangentially fed liquid stream $\left(E_{i n}\right)$ is equal to energy flux $\left(E_{d i s}\right)$ dissipated by tangential component of velocity:

$$
E_{\text {in }}=E_{\text {dis }} .
$$

This balance applies to tangential motion only. Losses of energy related to radial motion are covered by gravity force component resulting from slope of free surface of water. Liquid stream of density $\rho$ and discharge $Q$ fed to the system by a pipe of diameter $d_{\text {in }}$ (Fig. 4) delivers a kinetic energy flux described by the relation: 


$$
E_{\text {in }}=\rho Q \frac{v_{i n}^{2}}{2}=\frac{8 \rho Q^{3}}{\pi^{2} d_{i n}^{4}} .
$$

Intensity of mechanical energy losses related to unit volume of liquid (function " $S$ ") results from entropy balance law and is expressed as a sum of tensor $[D]$ coordinates of deformation velocity to the second power (e.g. Serrin 1959):

$$
S=2 \mu_{T}[D]:[D]
$$

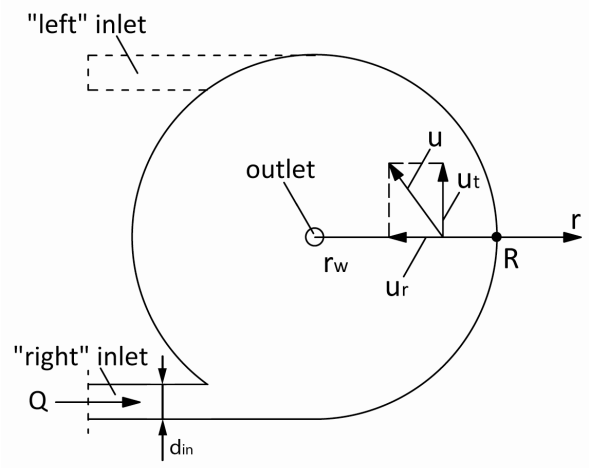

Fig. 4. Schematic diagram of a rotational separator

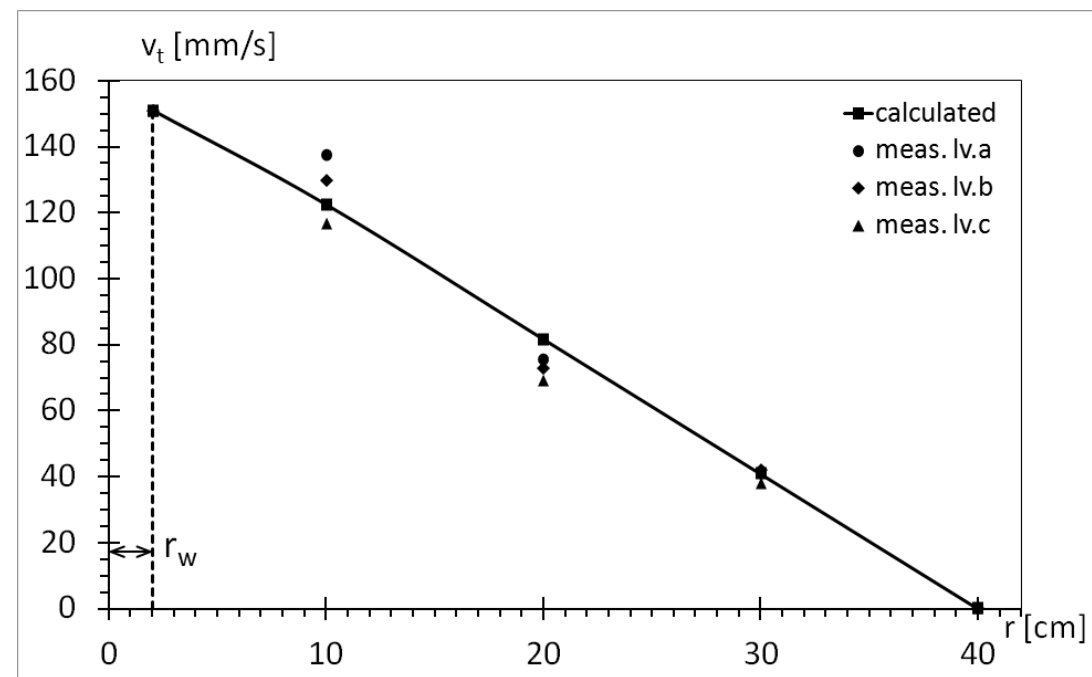

Fig. 5. Tangential velocity profile in rotational separator; a lv. $-10 \mathrm{~cm}$ below water surface; b lv. - middle depth; c lv. $-10 \mathrm{~cm}$ above bottom 
Therefore, total dissipated energy flux in the system of volume $V$ is equal to:

$$
E_{d i s}=2 \int_{V} \mu_{T}[D]:[D] d V .
$$

As area of the system in question is geometrically simple, only one coordinate of tensor $[D]$ is different from zero (Slattery 1999):

$$
D_{r t}=\frac{1}{2} r \frac{\partial}{\partial r}\left(\frac{u_{t}}{r}\right)
$$

After performing necessary transformations and calculations one obtains:

$$
A=6.14 Q\left(H R^{4} d_{i n}^{4}\right)^{-1 / 3} .
$$

In such type of devices, radial velocity is usually averaged vertically along tank depth $H$ and written as follows:

$$
u_{r}(r)=\frac{Q}{2 \pi r H} .
$$

However, existing disproportion between inlet diameter $\left(d_{i n}\right)$ and water outflow layer thickness $\Delta H$ (Fig. 7) prevents active radial flow from entirely filling water depth $H$. Taking into account the requirement for formally simple models, relation (18) was updated with assumed active layer thickness $H_{A}$. Analysis of measurement results (Fig. 6) allowed to accept the relation:

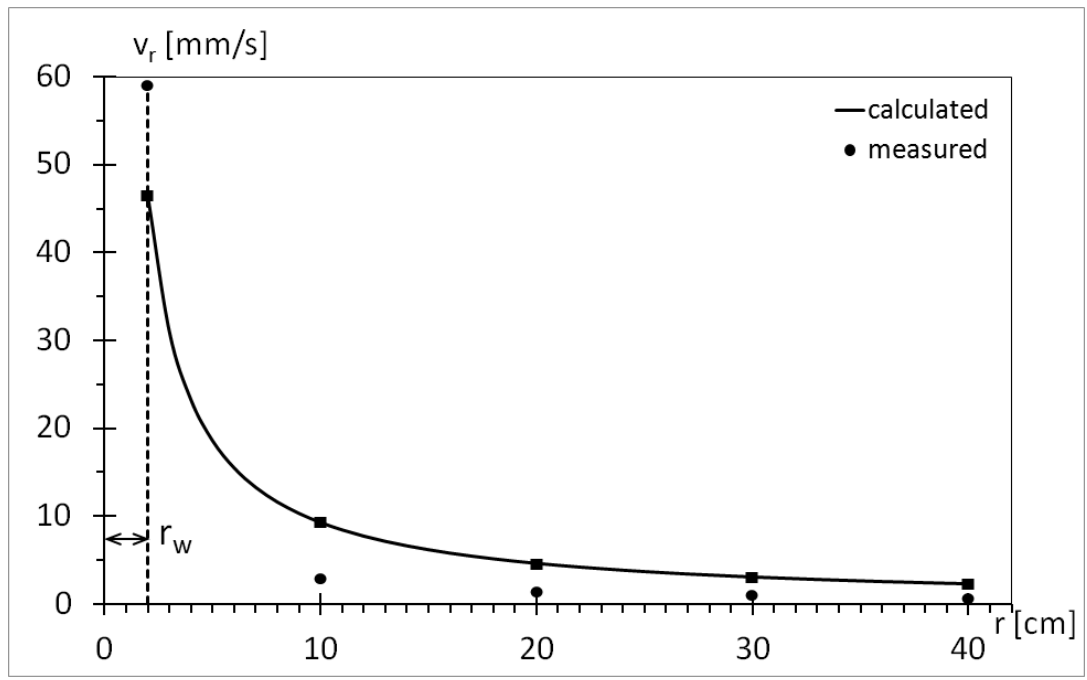

Fig. 6. Radial velocity profile in rotational separator 


$$
H_{A}=\frac{d_{\text {in }}+\Delta H}{2} .
$$

Finally, expression for radial velocity distribution acquires the form:

$$
u_{r}(r)=\frac{Q}{\pi r\left(d_{i n}+\Delta H\right)} .
$$

\subsection{Pressure Distribution in a Rotational Separator}

Pressure distribution in rotational separators is derived from the fundamental law of conservation of linear momentum. The equation of momentum conservation for cylindrical coordinates has the following form (Slattery 1999) (in this case radial component of mass force is equal to zero):

$$
\begin{gathered}
\rho\left(\frac{\partial u_{r}}{\partial t}+u_{r} \frac{\partial u_{r}}{\partial r}+\frac{u_{\theta}}{r} \frac{\partial u_{r}}{\partial \theta}-\frac{u_{t}^{2}}{r}+u_{2} \frac{\partial u_{r}}{\partial z}\right)=-\frac{\partial p}{\partial r}+\mu\left[\frac{\partial}{\partial r}\left(\frac{1}{r} \frac{\partial}{\partial r}\left(r u_{r}\right)\right)+\right. \\
\left.+\frac{1}{r^{2}}\left(\frac{\partial^{2} u_{r}}{\partial \theta^{2}}-\frac{2}{r^{2}} \frac{\partial u_{t}}{\partial \theta}+\frac{\partial^{2} u_{r}}{\partial z^{2}}\right)\right] .
\end{gathered}
$$

For an axially-symmetrical flow and a linear model of tangential velocity, the abovementioned equation obtains the form:

$$
\frac{\partial p}{\partial r}=\rho u_{r} \frac{\partial u_{r}}{\partial r}=\frac{\rho u_{t}^{2}}{r},
$$

where the tangential velocity is given by Eq. (11), radial velocities by Eq. (20), and its vertical component looks as follows:

$$
\frac{\partial p}{\partial z}=-\rho g
$$

By combining Eq. (11), (20), (22) and (23), one obtains:

$$
\left\{\begin{array}{l}
\frac{\partial p}{\partial r}=\frac{\rho Q^{2}}{4 \pi^{2} H r^{3}}+\frac{\rho A^{2}(R-r)}{r} \\
\frac{\partial p}{\partial z}=-\rho g
\end{array}\right.
$$

After a partial integration, formulas for pressure distribution along the chamber radius and vertical axis are obtained:

$$
\left\{\begin{array}{l}
p=-\frac{\rho Q^{2}}{8 \pi^{2} H r^{2}}+\rho A^{2}\left[R^{2} \ln r-2 R r=\frac{1}{2} r^{2}\right]+C_{1}(z) \\
p=-\rho g z+C_{2}(r)
\end{array}\right.
$$


where $C_{1} \neq f(r)$ and $C_{2} \neq f(z)$.

A transformation of Eq. (25) yields the formula for pressure distribution in a rotational separator:

$$
p=-\rho g z-\frac{\rho Q^{2}}{8 \pi^{2} H^{2} r^{2}}+\rho A^{2}\left[R^{2} \ln r-2 R r+\frac{1}{2} r^{2}\right]+C
$$

with the following boundary condition:

$$
r=R, \quad z=H=H_{\text {out }}+\Delta H, \quad p=p_{a},
$$

where $H_{\text {out }}$ and $\Delta H$ are the depths of the water layer in the chamber as shown in Fig. 7.

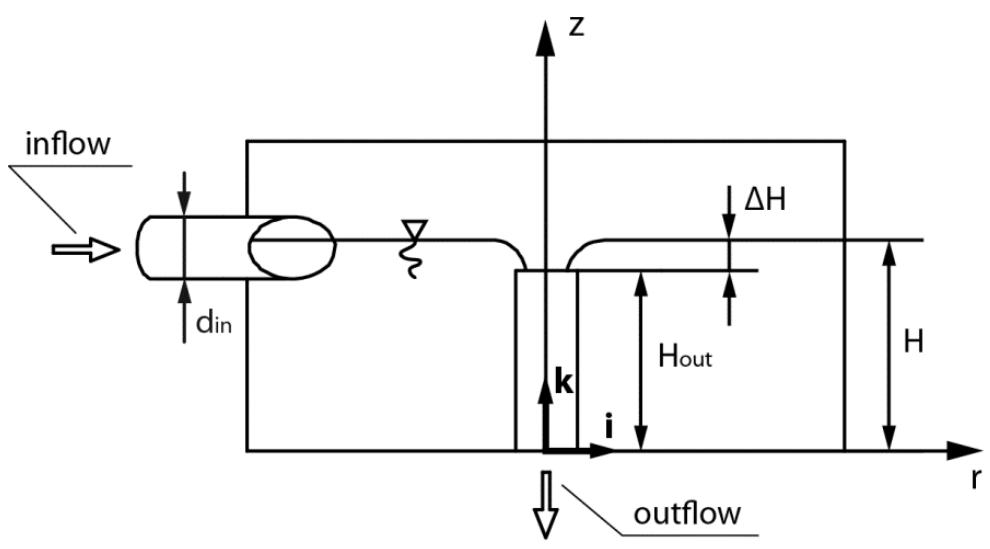

Fig. 7. Relations between different water depths at various points within the separator chamber

Applying boundary conditions (Eq. (27)) to Eq. (26), one can determine the integration constant $C$ and obtain the final formula for pressure distribution:

$$
\begin{gathered}
p(r, z)=p_{0}+\rho g(H-z)-\frac{\rho Q^{2}}{8 \pi^{2} H^{2}}\left(\frac{1}{r^{2}}-\frac{1}{R^{2}}\right)+ \\
+\rho A^{2}\left[R^{2} \ln \frac{r}{R}+2 R(R-r)-\frac{1}{2}\left(R^{2}-r^{2}\right)\right] .
\end{gathered}
$$

Using Eq. (6) and Eq. (28), an expression describing the pressure force is obtained:

$$
F_{T P}=-\frac{\rho Q^{2} V}{4 \pi^{2} H^{2} r^{3}}-\rho A^{2} V \frac{(R-r)^{2}}{r} .
$$




\subsection{Normalization of Forces Important to Rotational Separator Design}

The Substitution of Eq. (11) into Eq. (2) and Eq. (5) yields expressions for the drag and centrifugal forces:

$$
\begin{gathered}
F_{D N}=-C_{D} S_{c} \frac{\rho Q^{2}}{\pi^{2} r^{2}\left(d_{i n}+\Delta H\right)}, \\
F_{o}=\rho_{c} V A^{2} \frac{(R-r)^{2}}{r} .
\end{gathered}
$$

In order to present the variability of forces important to rotational separator design in a manner that is independent of system characteristics, relations given by Eq. (25), (30) and (31) were transformed. Normalized forces are a function of only the relative distance from the chamber axis $(r / R)$. The pressure force is a sum of two components - one depending on the tangential velocity $\left(F_{T P T}\right)$, the other - on the radial velocity $\left(F_{T P R}\right)$. The relations are presented in such a way to show relative distribution of particular forces. As mentioned in Section 2.2, practical method of separator design should be based on set of forces acting on characteristic particle of the suspension.

The normalized versions of relations describing the specific forces are as follows:

$$
\begin{gathered}
\alpha_{o}=\frac{F_{0}}{\rho_{c} \mathrm{~A}^{2} V R}=\left(\frac{R}{r}-2+\frac{r}{R}\right), \\
\alpha_{D N}=\frac{F_{D N} \pi R^{2}\left(d_{i n}+\Delta H\right)}{\rho Q^{2} S_{c}}=-\frac{R^{2}}{r^{2}}, \\
\alpha_{T P T}=\frac{F_{T P T}}{\rho \mathrm{A}^{2} V R}=-\left(\frac{R}{r}-2+\frac{r}{R}\right), \\
\alpha_{T P R}=\frac{F_{T P R} 4 \pi^{2} H^{2} R^{3}}{\rho Q^{2} V}=-\frac{R^{3}}{r^{3}} .
\end{gathered}
$$

It can be seen that the normalized versions of the forces $\mathbf{F}_{\mathbf{0}}$ and $\mathbf{F}_{\text {TPT }}$ are identical functions of the $r$ coordinate (Eq. (32) and (34)). Figs. 8, 9, 10 present the normalized distributions of the abovementioned forces in a rotational separator. All acting forces reach their extreme values in the central part of the separator, the fact leading to possible conception of the design method: in a properly designed rotational separator maximum value of the centrifugal force acting on a particle (pushing the particle far outwards) is greater than the sum of all remaining forces (tending to remove the particle from the chamber). 


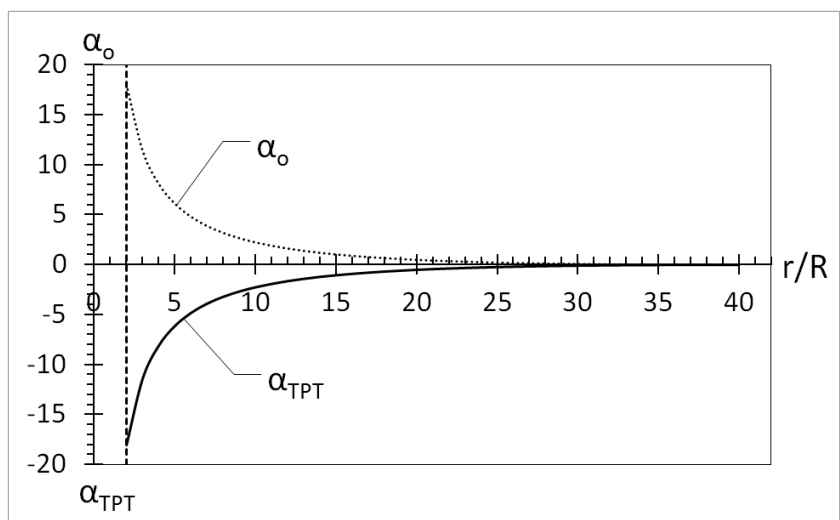

Fig. 8. Normalized distribution of the centrifugal force and the tangential component of the pressure force

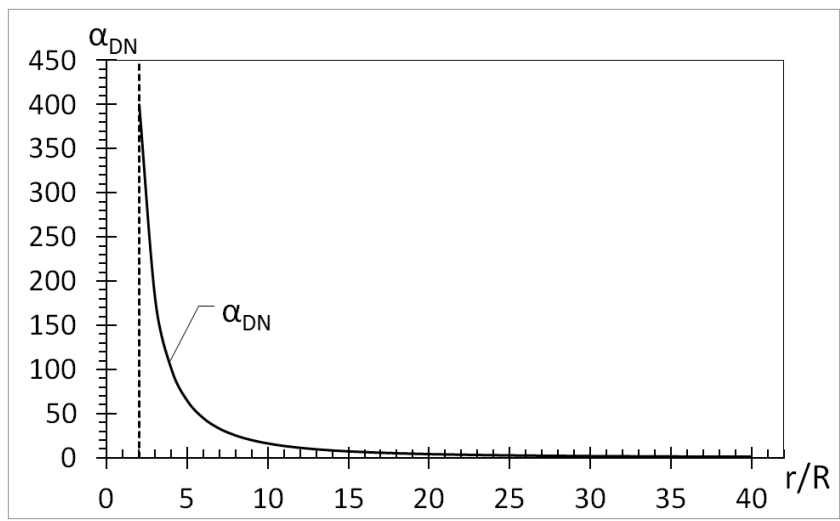

Fig. 9. Normalized distribution of the stress force

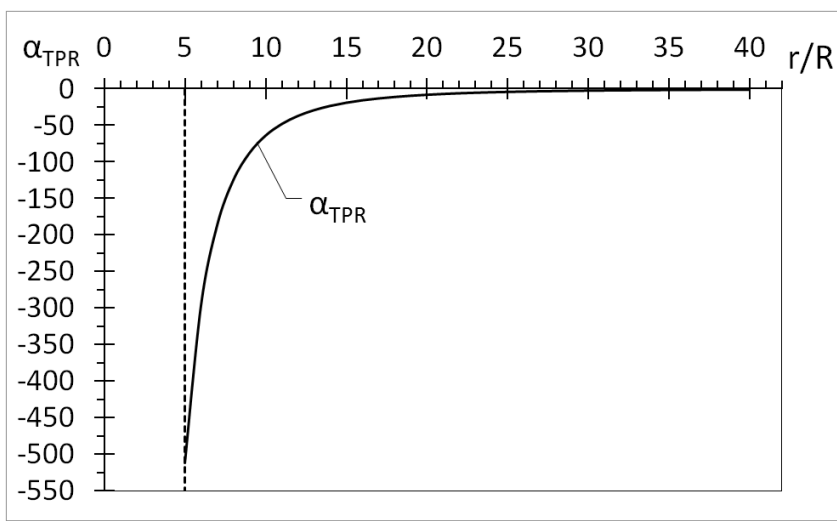

Fig. 10. Normalized distribution of the radial component of the pressure force 


\section{Conclusions}

With the growing need for storm water treatment devices, there is a potential for the application of rotational separators on an industrial scale. Until now, the phenomenon of centrifugal force has mainly been exploited in cyclones for dust removal from air. However, the rotational motion of water flow can also be effectively used to remove suspensions from waste water. As no adequate method of designing separators has been developed so far, the ultimate aim of the research being conducted is to arrive at a method that could be safely used to design rotational separators, a method that is both mathematically simple and physically well-grounded. The specification of forces acting on a suspended particle is an important step in the development of such a design method.

\section{Acknowledgement}

The paper has been financially supported by the Polish National Centre of Science (grant N N 523554 738).

\section{References}

Bernardo S., Martignoni W. P., Quintani C. L. (2007) Evaluation of cyclone geometry and its influence on performance parameters by CFD, Braz. Jour. Chem. Eng., 24, 1-17.

Gronowska M., Sawicki J. M. (2011) Study of rotational separators operation and design, [in:] Technical progress in sanitary engineering, Wydawnictwo Politechniki Gdańskiej, Gdańsk, 40-48.

Osmólska-Mróz B. (1996) Principles of environmental protection for design, construction and maintenance of roads, Section 7: Protection of waters surrounded by roads, Instytut Dróg i Mostów, Warszawa (in Polish).

PN-S-02204 (1997) Drogi samochodowe. Odwodnienie dróg. (Polish standard)

Serrin J. (1959) Mathematical principles of classical fluid mechanics, Handbuch der Physik, Band VIII/1. Berlin-Goettingen-Heidelberg.

Slattery J. C. (1999) Advanced transport phenomena, University Press, Cambridge.

Smith J. L. (1959) Experimental and Analytical Study of the Vortex in the Cyclone Separator, MIT, Cambridge.

Soo L. (1996) Fluid Dynamics of Multiphase Systems, Blaisdell Publ. Comp., London.

Stairmand C. J. (1951) The design and performance of cyclone separators, Trans. Inst. Chem. Eng., 29, 356-373.

Trawinski H. (1969) Practical aspects of the design and industrial applications of the hydrocyclones, Filtr. and Sep., 6, 361-369.

Warych J. (1998) Gas Purification, WNT, Warszawa (in Polish).

Wikipedia, the Free Encyclopedia (2010) Cyclonic separation, http://en.wikipedia.org/wiki/Cyclonic_separation. 